\title{
Bilan dosimétrique 1999 dans les centrales nucléaires d'EDF : commentaires et analyses
}

\author{
Y. GARCIER*, Ph. HARTMANN**
}

(Manuscrit reçu le 30 juin 2000, accepté le 9 juillet 2000)

RÉSUMÉ L'année 1999 se solde par un bilan plutôt positif en ce qui concerne la valeur de la dose des travailleurs qui est en constante diminution. L'objectif fixé pour l'an 2000 (1,2 H.Sv par réacteur) est d'ores et déjà atteint en 1999 (1,17 H.Sv). Toutefois, si le palier de 1300 MWe progresse encore, le palier de 900 MWe semble marquer le pas. Pour ce qui est de la dosimétrie individuelle, si celle-ci est restée stable en moyenne, il n'en demeure pas moins que le nombre de travailleurs les plus exposés a fortement baissé. Ces progrès sont à mettre sur le compte des actions engagées dans le cadre de l'optimisation mais aussi de la réduction de la durée des arrêts pour rechargement, de l'allongement du cycle de combustible (chargement un quart de cour) et de la révision de la politique de maintenance (mise en ouvre de l'optimisation de la maintenance fiabilisée). L’année 1999 a vu les premiers effets du plan «propreté» lancé en 1998. Ce plan a déjà permis d'améliorer notablement la situation en matière de contamination des transports de combustible usé. Toutefois, l'incident d'exposition survenu à Tricastin ainsi que d'autres dysfonctionnements mineurs nous appellent à la vigilance et à plus de rigueur dans l'exécution des opérations liées à la radioprotection.

ABSTRACT 1999 occupational dosimetry in EDF's nuclear power plants: comments and analyse.

The year 1999 ended with rather positive results concerning worker dosimetry, which is constantly decreasing. The targeted objective for the year 2000 (1.2 man.Sv per reactor) was already attained in 1999 (1.17 man.Sv). However, even though the $1300 \mathrm{MWe}$ level still continues to progress, the $900 \mathrm{MWe}$ level seems to marking time. In the case of individual dosimetry, even though it remained stable on the whole, nonetheless the number of workers the most strongly exposed decreased very much. These advancements should be considered as a part of the actions committed not only within the scope of optimization, but also in the reduction of shutdown periods for refueling, the extension of the fuel cycle (loading $1 / 4$ of a core) and the revision of the maintenance policy (implementation of the optimization of reliability oriented maintenance). 1999 was the year when the initial effects of the "cleanliness" plan launched in 1998 were noted. This plan has already allowed improving notably the situation in terms of contamination in the transport of spent fuel. However, the exposure incident which occurred at Tricastin, as well as other minor malfunctions, incite in us prudence and more rigor in the performance of operations related to radiation protection.

\footnotetext{
* EDF, Pôle industrie Cap Ampère, 1 place Pleyel, 93282 St-Denis Cedex, France.

** EDF, Service de radioprotection, 22-28 rue Joubert, 75009 Paris, France.
} 


\section{Doses collectives}

Le parc de production nucléaire d'EDF comprend 58 réacteurs à eau sous pression (REP) répartis sur 19 sites (34 réacteurs de $900 \mathrm{MWe}, 20$ réacteurs de $1300 \mathrm{MWe}$, 4 réacteurs de $1450 \mathrm{MWe}$ dont un encore en phase de démarrage).

La dose collective moyenne, par réacteur, reçue par l'ensemble des personnes exposées a diminué de $5 \%$ passant de 1,2 H.Sv en 1998 à 1,17 H.Sv en 1999. Cette valeur est légèrement inférieure à l'objectif qui avait été fixé pour l'an 2000 , à savoir $1,2 \mathrm{H} . \mathrm{Sv}$. Elle est le résultat de la poursuite des efforts engagés par l'entreprise pour optimiser la dosimétrie, notamment sur les grosses opérations de maintenance.

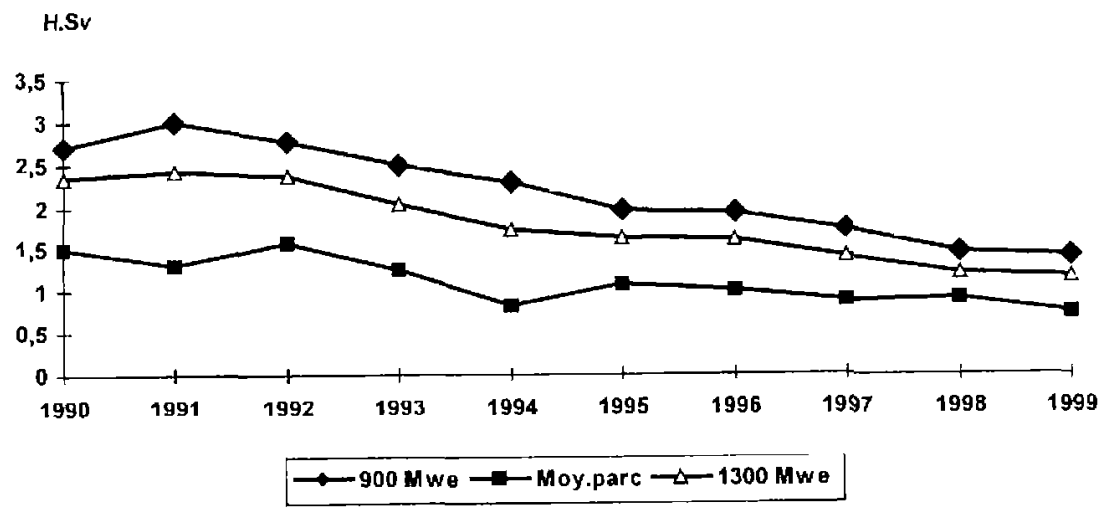

Fig. 1. -Évolution des doses collectives (doses moyennes annuelles REP).

Evolution of collective doses (mean annual doses PWR).

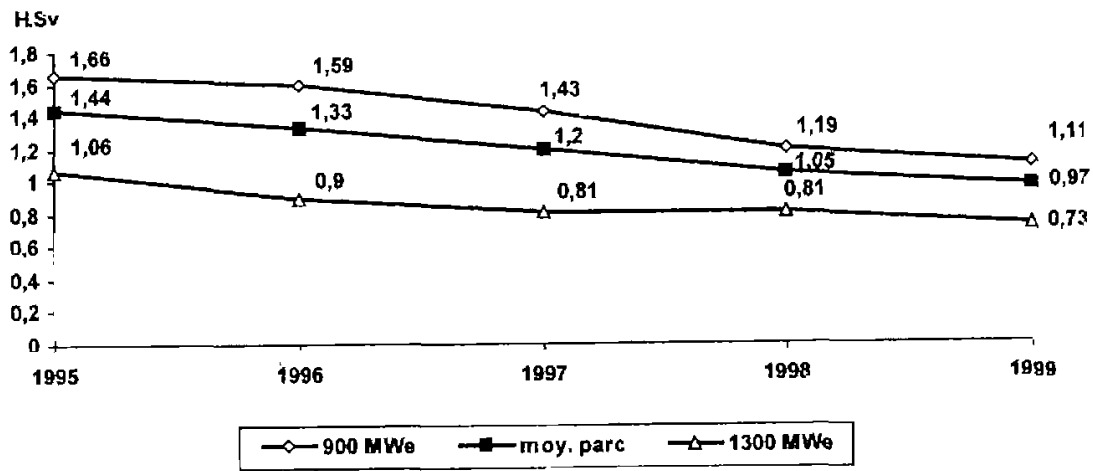

Fig. 2. -Doses collectives moyennes lors des arrêts de courte durée (ASR, visites partielles). Mean collective doses during short shutdowns (ASR, partial inspections). 


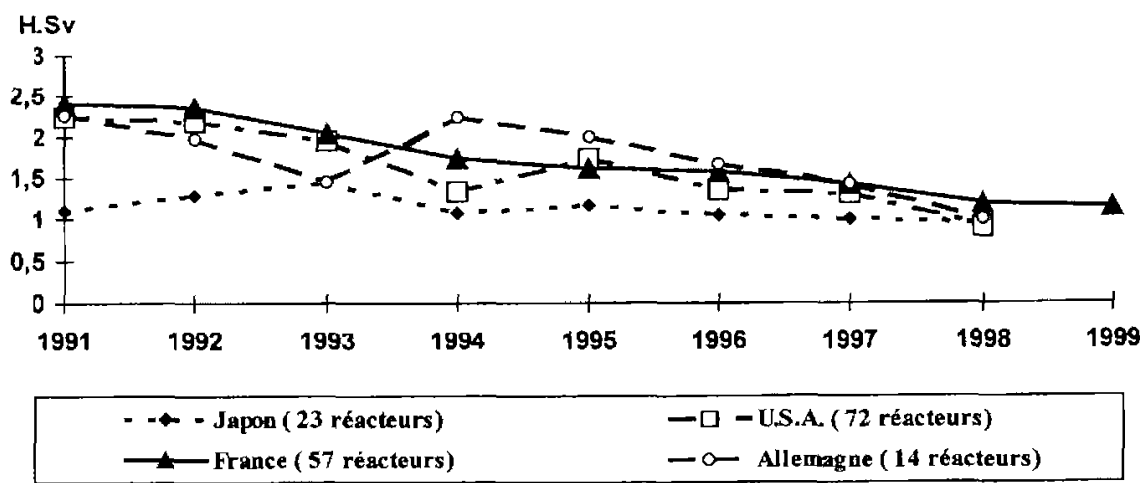

Fig. 3. -Comparaison internationale (source OCDE/ISOE, 1998). Doses moyennes annuelles collectives par réacteur REP (réacteurs à eau sous pression).

International comparison (source OECD/ISOE, 1998). Average annual collective doses per reactor PWR (pressurized water reactor).

Cette valeur moyenne masque toutefois des écarts entre les tranches des différents paliers de puissance, même si ces écarts se resserrent ; les « meilleures» tranches d'un point de vue dosimétrique ayant un effet d'entraînement sur les «moins bonnes».

Les moyennes pour les tranches de $900 \mathrm{MWe}$ et de $1300 \mathrm{MWe}$ sont respectivement de 1,42 H.Sv (1,45 en 1998) et $0,73 \mathrm{H} . \mathrm{Sv}(0,91$ en 1998).

La figure 1 situe les résultats d'EDF année par année depuis 1990.

Pour le palier de $900 \mathrm{MWe}$, la dosimétrie collective semble marquer le pas. Sur le palier de 1300 MWe la diminution de la dose collective est sensible. Elle s'explique non seulement par les efforts réalisés pour réduire les doses sur les chantiers à risque, mais aussi par la volonté de réduire le volume des opérations effectuées en zone contrôlée. La généralisation des arrêts à simple rechargement (ASR), l'allongement des cycles du combustible sur le palier de $1300 \mathrm{MWe}$ ainsi que la mise en place d'une politique de maintenance fiabilisée ont permis d'agir dans ce sens (Fig. 2).

\section{Comparaisons internationales}

Les statistiques internationales montrent que la dose collective moyenne par réacteur REP continue de baisser dans les grands pays de l'industrie nucléaire (Fig. 3). Cette progression est plus sensible au Japon, aux Etats-Unis et en Allemagne qu'en France. Mais pour être tout à fait pertinente, cette comparaison devrait tenir compte des politiques adoptées par ces pays en matière de gestion du combustible (longueur du cycle) et de maintenance, car ces paramètres influencent directement la dosimétrie. 
mSv

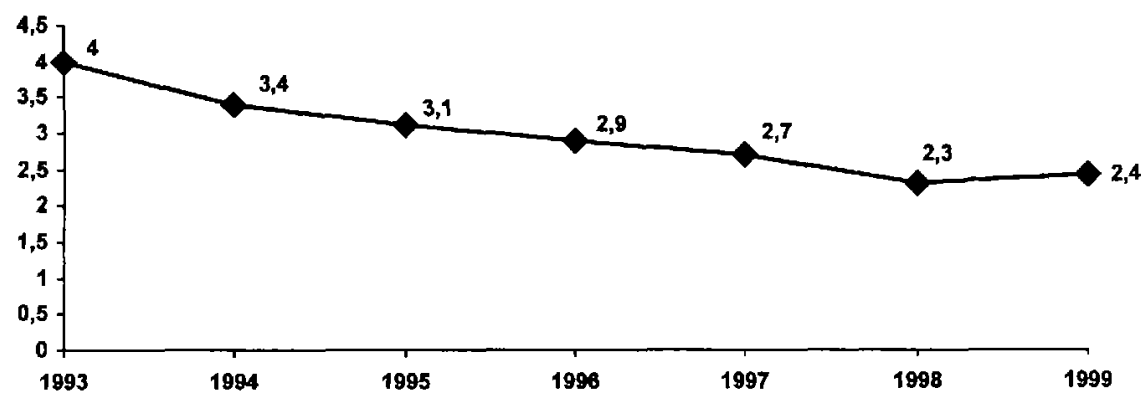

Fig. 4. -Dose individuelle annuelle moyenne EDF + prestataires.

Average annual individual dose EDF + providers.

On notera que pour des grosses opérations de maintenance telles que le remplacement de générateurs de vapeur (RGV), EDF obtient de bien meilleurs résultats dosimétriques. À titre d'exemple, l'opération réalisée à Tricastin, en 1999, s'est traduite par une dosimétrie collective de $0,2 \mathrm{H}$.Sv par générateur de vapeur (GV) alors qu'elle est d'environ 0,5 à $0,8 \mathrm{H}$.Sv/GV aux États-Unis. Les méthodes et pratiques développées par EDF pour des opérations lourdes s'avèrent donc efficaces en matière de dosimétrie. Celles-ci pourraient être utilement transposées sur d'autres chantiers de maintenance et mises à profit pour réduire les doses.

\section{Doses individuelles}

En 1999, le nombre de travailleurs exposés (ayant reçu une dose supérieure ou égale au seuil d'enregistrement: $0,01 \mathrm{mSv}^{(1)}$ ) a été de $\mathbf{3 2} 500$ personnes (15300 EDF ; 17200 prestataires). Le nombre de travailleurs exposés dont la dose annuelle est inférieure au seuil d'enregistrement est de $\mathbf{5} \mathbf{1 3 6 .}$

Les statistiques dosimétriques prises en compte pour suivre l'évolution des progrès réalisés portent sur le nombre de travailleurs ayant reçu dans l'année une dose supérieure au seuil d'enregistrement. Ils étaient 27340 dont 14900 prestataires, en 1999.

Sur ces bases, la valeur moyenne annuelle des expositions individuelles est restée stable pour les travailleurs exposés dont la dose est strictement supérieure au seuil d'enregistrement : elle est de 2,35 mSv en 1999 (2,30 mSv en 1998). La dose

1 Le seuil d'enregistrement recommandé par la Commission internationale de protection radiologique (CIPR) est de $0,5 \mathrm{mSv}$. 


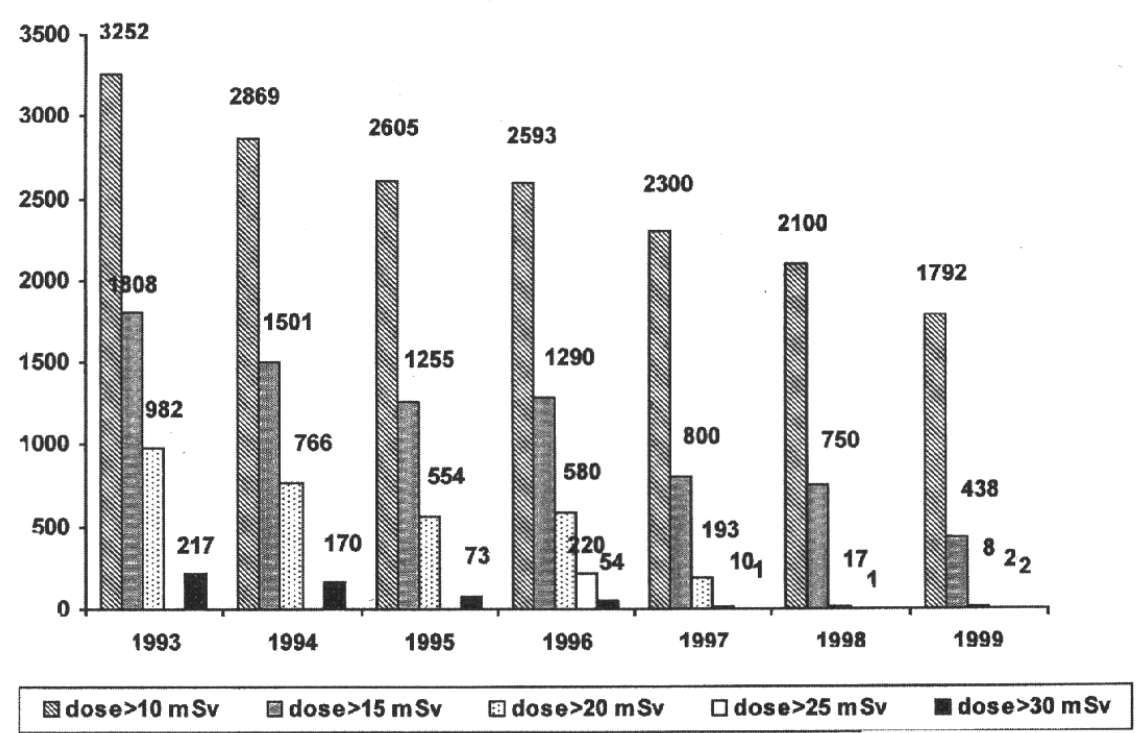

Fig. 5. - Nombre d'intervenants (EDF + prestataires) ayant dépassé le niveau de dose annuelle indiqué.

Number of intervening parties (EDF + providers) who exceeded the indicated annual dose level.

moyenne annuelle est de 3,36 mSv pour les travailleurs d'entreprises extérieures (3,24 $\mathrm{mSv}$ en 1998) et de 1,15 mSv pour les agents d'EDF (1,16 en 1998). Cette stabilité s'explique par le fait que les travailleurs exposés ont été moins nombreux en 1999 (27 340) qu'en 1998 (28 392), soit une diminution de 1052 (4 \%). La dose moyenne n'a donc pas changé bien que la dose collective ait diminué.

La figure 4 montre l'évolution des doses des travailleurs ayant reçu une dose supérieure au seuil d'enregistrement.

L'inflexion dans la courbe d'évolution de la dosimétrie individuelle moyenne ne doit pas nous faire oublier les actions engagées en matière d'optimisation des doses, notamment au travers de la charte de progrès signée en 1997 avec les principales entreprises prestataires intervenant sur les sites nucléaires d'EDF. Ces actions concrètes se sont révélées efficaces pour réduire la dosimétrie des travailleurs les plus exposés comme le montre la figure 5 qui traduit bien une réduction des effectifs dans toutes les classes de doses supérieures à $10 \mathrm{mSv}$. Ces derniers ne représentent en fait qu'environ $6 \%$ des travailleurs exposés.

On notera aussi que le nombre de travailleurs ayant reçu une dose annuelle supérieure à $20 \mathrm{mSv}$ a été ramené à un niveau très faible en 1999. Huit personnes ont dépassé cette valeur en 1999 contre 17 en 1998 et 193 en 1997. Hormis deux dépassements 
nets de cette valeur (incident de Tricastin, $340 \mathrm{mSv}$ et de Dampierre, $30 \mathrm{mSv}$ ), les six autres cas n'excèdent pas $21 \mathrm{mSv}$.

\section{Incidents de personnes en 1999}

Le 11 mars 1999, un technicien de la centrale de Tricastin a reçu une dose de $340 \mathrm{mSv}$ en pénétrant dans le puits de cuve (zone contrôlée classée «rouge») alors que la centrale était à l'arrêt pour maintenance. En raison du dépassement de la limite réglementaire fixée pour les travailleurs à $50 \mathrm{mSv}$ par an, cet incident a été classé au niveau 2 de l'échelle INES.

Le 29 mars 1999 à Chinon : l'entreposage de matériel irradiant dans la zone contrôlée de l'atelier des matériaux irradiés (AMI) a induit un débit de dose dans les bureaux (zone conventionnelle) situés au-dessus de cette zone. Quatre personnes DATR ont reçu chacune des doses comprises entre 2 et $5 \mathrm{mSv}$. L'incident a été classé au niveau 1 de l'échelle INES.

Le 8 septembre 1999 à Gurcy-le-Chatel : la disparition d'une source de neutrons (11 GBq), utilisée pour faire des expériences au centre de formation professionnelle, a été constatée. Elle a été retrouvée dans un puisard quatre jours plus tard. Cette disparition est vraisemblablement due à un acte de malveillance. Une plainte a été déposée par le centre. Cet événement aurait pu avoir des conséquences sérieuses si quelqu'un avait été en contact direct et prolongé avec la source. Ceci nous appelle à la vigilance et nous conduit à nous interroger sur le mode de gestion des sources radioactives utilisées en centrales nucléaires mais aussi dans les autres installations d'EDF.

Le 24 septembre 1999 à Cattenom : l'absence de balisage au voisinage d'un couvercle de cuve usagé, entreposé sur le site en attente d'évacuation, a conduit les Autorités à dresser procès-verbal pour infraction à la réglementation. L'incident a été classé au niveau 1 de l'échelle INES.

Enfin, il faut noter quelques évacuations de bâtiment réacteur (BR) à la suite de déclenchements de balise de surveillance de la radioactivité sur les chantiers. Bien que ces événements n'aient pas eu d'incidence sur le plan sanitaire (les examens anthropogammamétriques pratiqués sur les personnes évacuées n'ont pas révélé de contamination significative), leur retentissement dans les médias a été grand. À Cruas, la DSIN a estimé que l'évacuation s'est faite tardivement et, pour cette raison, l'incident a été classé au niveau 1 de l'échelle INES.

\section{Conclusion}

Les efforts entrepris de longue date, par EDF, dans le domaine de la radioprotection ont porté leurs fruits. Pour ce qui est des travailleurs, qu'ils soient d'EDF ou 
d'entreprises prestataires, les doses collectives et individuelles n'ont cessé de baisser grâce à la démarche d'optimisation «ALARA", lancée en 1991 ( $-60 \%$ en terme de dose collective moyenne par réacteur). Le niveau de radioprotection est globalement satisfaisant. Pourtant, ce bilan recèle quelques incidents qui ont révélé un manque de rigueur dans l'exécution de certaines opérations (transport de combustible usé...). Ces incidents n'ont toutefois pas eu de conséquence sur la santé du public et des travailleurs.

EDF a pris conscience de cette situation et a décidé d'agir en mettant sur pied un plan d'action dont la priorité est le renforcement de la gestion de la radioprotection tant au niveau national que local.

\section{RÉFÉRENCES}

EDF (1999) Rapport d'activité sur la radioprotection.

OCDE/ISOE (1998) Occupational exposures at nuclear power plants. $8^{\circ}$ rapport annuel, OCDE/AEN. 